\title{
Classification of Imaging Phenotypes of Heart Failure with Preserved Ejection Fraction (HFpEF) on MRI
}

\author{
Atul Kapoor*, Aprajita Kapoor, Goldaa Mahajan \\ Department of Radiology, Advanced Diagnostics and Institute of Imaging, Amritsar, Punjab, India \\ Email address: \\ masatulak@aim.com (A. Kapoor) \\ ${ }^{*}$ Corresponding author \\ To cite this article: \\ Atul Kapoor, Aprajita Kapoor, Goldaa Mahajan. Classification of Imaging Phenotypes of Heart Failure with Preserved Ejection Fraction \\ (HFpEF) on MRI. Cardiology and Cardiovascular Research. Vol. 4, No. 4, 2020, pp. 187-195. doi: 10.11648/j.ccr.20200404.14
}

Received: October 3, 2020; Accepted: October 15, 2020; Published: October 21, 2020

\begin{abstract}
Objective: The purpose of this study was to define imaging phenotypes of patients of HFpEF by the use of Cardiac MR techniques after initial evaluation with color Doppler examination. HFpEF is a common type of heart failure in the middle age with a high 5 year mortality. Diagnosis of HFpEF is based on combination of findings of colour Doppler echocardiograpy and serum NT- pro BNP levels. So far it has not been possible to identify imaging phenotypes of HFpEF which can improve patient management by use of imaging. Methods: Retrospective study of 50 patients of HFpEF who were investigated by cardiac MR using T1 mapping, extracellular estimation of left and right ventricle along with feature tracking to determine the systolic and diastolic strain of both the left and right ventricle. All patients were classified into three groups on the basis of findings observed. Results: The study showed three groups of patients. 21 patients in group I were HFpEF-iLV type with reduced GCSe'r alongwith increased E/GCSe'r values and showed no structural change. Group II (HFpEF -IpcPH type) had 13 patients which showed reduced EGCSe'r of left ventricle alongwith mild increased LAVI but also had reduced GCS of right ventricle with no structural change in latter. Group III (HFpEF-CPCPH) had 16 patients which alongwith findings of group III showed increased ECV of right ventricle. Strong correlation of E/GCSe'r was seen with proBNP levels in group III patients and with right ventricle ECV. E/GCSe'r was a strong marker in all the three groups with right ventricle ECV to decide the phenotype of patient. Conclusion: CMR was found to be useful in categorizing imaging phenotypes of HFpEF which can have a strong bearing in the management of such patients.
\end{abstract}

Keywords: HFpEF, Cardiac MR, Diastolic Dysfunction, Extracellular Volume

\section{Introduction}

$\mathrm{HFpEF}$ is a complex syndrome resulting from impaired ventricular filling and is a common cause of hospital admission after age of 65 years with a 5 year mortality of $43.2 \%[1,2]$. The diagnosis of HFpEF is based on combination of factors as suggested by European society of cardiology (ESC) 2019 [3] i.e. it is a composite of pretest clinical probability, echocardiographic parameters and natriuretic peptide blood levels. Based on these parameters a score of more than 5 establishes the diagnosis while less than 1 is negative for $\mathrm{HFpEF}$ and a intermediate score of 2-4 requires further evaluation. Even after forming the diagnosis further testing is required to determine its etiology. The etiology of HFpEF typically evolves from a combination of risk factors and comorbidities, including advanced age, female sex, obesity, systemic arterial hypertension, diabetes mellitus, renal dysfunction, anaemia, iron deficiency, sleep disorders, and chronic obstructive pulmonary disease. HFpEF is also seen to have different stages of disease depending on etiology and disease progress. Hence a better classification of $\mathrm{HFpEF}$ is recommended by ESC [3]. Samson etal [4] classified clinical phenotypes of this syndrome based on etiological factors. However this classification may not be of use when planning treatment for such patients as more than one factor may be coexisting in these patients. Pasha etal [5] recently described the role of cardiac MR (CMR) in the evaluation of left ventricular diastolic function and suggested that CMR has a unique position in not only accurately detecting structural changes but also functional changes in heart in HFpEF. This retrospective study was therefore designed to classify known patients of HFpEF based on 
imaging phenotypes using cardiac MR.

\section{Material and Methods}

This retrospective study comprised of imaging data of 50 known patients of heart failure who had at least 5 point HFpEF score as per ESC guidelines 2019 [3] and underwent imaging evaluation at our institute over a period of last two years. Findings of all patients including clinical examination, EKG and color and tissue doppler echocardiography along with routine blood profile including NT-pro BNP levels were reviewed along with findings of Cardiac MRI. CMR was done on a 1.5 Tesla Cardiac MRI scanner (Amira, Siemens, Shenzen, China) using a 16 channel phased array surface coil with EKG gating. Cine images of the heart were taken from base to the apex in short axis, 2 chamber longitudinal axis and 4 chamber views. Pre contrast T1 maps of the heart were obtained in the mid, basal and apical short axis views using Modified Look-Locker inversion recovery sequence (MOLLI). A perfusion study was then done in short axis views at the three sites as described using Intravenous $0.2 \mathrm{mmol} / \mathrm{Kg}$ gadopentetate dimeglumine contrast (Magnevist, Bayer Healthcare, Germany) bolus injection. Post contrast T1 maps were obtained in the similar positions as the plain study at 10 minutes interval. This was followed by Phase contrast inversion recovery sequence for LGE if any of the left ventricle from the base to the apex of left ventricle. 15 normal adults were enrolled as volunteers with no history of hypertension, diabetes or any prior heart disease for CMR examination to determine normal right and left ventricle CMR strain parameters in our population. (Table 1).

Table 1. Normal Reference values for three dimensional feature tracking CMR.

\begin{tabular}{llll}
\hline Sno & Parameter & Mean & Range \\
\hline 1 & GCS & -18 & -14 to -23 \\
2 & GLS & -12 & -11 to- 14 \\
3 & GCS' & -75 & -65 to 1.15 \\
4 & GCS e' & 0.75 & 0.65 to 1.10 \\
5 & GCSA' & 0.45 & 0.30 to 0.50 \\
6 & E/GCSe'r & $<0.75$ & $0.65-.85$ \\
\hline
\end{tabular}

* GCS: global circumferential strain, GLS: global longitudnal strain, GCS': global circumferential strain rate, GCSe': Global circumferential early diastolic strain rate.

\subsection{Image Analysis}

Was done by experienced cardiac radiologists to record for wall motion abnormalities of the left ventricle, areas of LGE enhancement, thickness of the myocardium and percentage of scar tissue. The pre and post contrast T1 maps were processed for ECV maps using (ECV maps software, Syngo Frontier, Siemens Germany and CMR Segment software, University of Lund, Sweden). Extracellular volumes of both right and left ventricle were estimated and a normal of $25 \%$ and $27 \%$ set as the threshold. Ventricle function analysis along with feature tracking was done on Strain analysis software (Siemens Germany). Ejection fraction of left and right ventricle, left ventricle mass, End diastolic volumes and wall thickness of right and left ventricle, left atrial volume index, global circumferential systolic strain, Global longitudinal strain, global circumferential diastolic strain, global circumeferential strain rate early and late diastolic for both right and left ventricle determined. E/GCSe' was also calculated for both right and left ventricle. The normal cut off values for the above para meters are listed in table 1. Based on the above parameters all patients of HFpEF were categorized into three imaging phenotype groups. Group I comprised of patients with HFpEF- isolated LV type (ilv) which had isolated diastolic left ventricle filling disturbances. Group II patients were those of HFPEF -isolated postcapillary pulmonary hypertension (ipcph)) with combined right and left ventricle diastolic filling abnormalities. Group III HFpEF patients had combined pre and post capillary pulmonary hypertension (Cpcph) and showed left and right ventricles diastolic abnormalities with increased ECV of right ventricle with or without reduced systolic strain of right ventricle.

\subsection{Statistical Analysis}

Was done using Analyse -IT software (Leeds UK) and mean values alongwith standard deviation and distribution of all continuous variables calculated and values compared for three groups using Kruskal Wallis test to deterimine the statistical significance of differences with $p$ value of less than 0.05 being significant. Pearson correlation test was also done between variables in all the three groups.

\section{Results}

Patient demographics are shown in (table 2). The mean age of patients of 61.8 years $(60-63.5$ years $95 \% \mathrm{CI})$. There were 43 females and 7 males. Mean left ventricle ejection fraction was $55.1 \%$ while of the right ventricle was $49.1 \%$. 21 patients were seen in Group I, 13 in group II and 16 in group III. The imaging variables of three groups are enlisted in table 2. Statistically significant differences which were seen in three groups in variables were in E/eGCSe'r, ECV of right ventricle, left atrial volume index, left ventricle global early and late diastolic strain rate.

Table 2. Baseline demographics of patients.

\begin{tabular}{llll}
\hline S. No & Character & Mean & 95\% CI \\
\hline 1 & Age & 61.3 & $60-63.8$ \\
2 & Sex & & \\
& Males & $43(86 \%)$ & \\
& Females & $7(14 \%)$ & \\
3 & Family history CAD & $9(18 \%)$ & \\
\hline
\end{tabular}




\begin{tabular}{llll}
\hline S. No & Character & Mean & 95\% CI \\
\hline 4 & History of atrial fibrillation & $6(12 \%)$ & \\
5 & BMI (Kg/m2) & 29.5 & $28.5-31.8$ \\
6 & History of hypertension & 28 & \\
7 & Diabetes mellitus & 13 & \\
8 & History of previous MI & 2 & 18 \\
9 & Dysnoea & 25 & $53.7-56.7$ \\
& NYHA II & 7 & $47.5-50.7$ \\
10 & NYHA III & 55.2 & $80-85.3$ \\
11 & Ejection fraction (\%) Left ventricle & 49.1 & $3.5-9.5$ \\
11 & Ejection fraction (\%) Right ventricle & 82 & $102-114$ \\
12 & End systolic volume index (m/m2) & 6.5 & $328-413$ \\
13 & End diastolic wall thickness (mm) & 109 & \\
14 & Left ventricle mass gm & 371 & \\
\hline
\end{tabular}

Table 3. Imaging variables in HFpEF on CMR.

\begin{tabular}{lllll}
\hline VARIABLE & GROUP I & GROUP II & GROUP III & p value \\
\hline LV E/GCSer & 1.01 & 1.2 & 2.1 & 0.001 \\
LVEF & 57.4 & 53.3 & 54.2 & 0.08 \\
LVECV & 25.6 & 29.1 & 29.3 & 0.11 \\
PRO-BNP & 260 & 314 & 468 & 0.4 \\
E/e & 12.8 & 14.4 & 13.3 & 0.76 \\
LVED & 89.1 & 91.5 & 84.9 & 0.25 \\
LV GCS & -18.1 & -16.48 & -13.8 & 0.005 \\
lV GCS & -14.1 & -11.48 & -11.3 & 0.96 \\
LVGCSE & 83.9 & 82.8 & 52.5 & 0.001 \\
LVGCSA & 68 & 48 & 34 & 0.001 \\
LAVI (ml/m2) & 26.4 & 30.38 & 34.7 & 0.001 \\
LVM & 113.1 & 113.3 & 103 & 0.87 \\
LVED & 89.1 & 91.5 & 84.9 & 0.25 \\
RVED & 81.1 & 87.3 & 88.2 & 0.11 \\
RVED & 81.1 & 87.3 & 88.2 & 0.11 \\
RVEF & 52.4 & 50.4 & 44.3 & 0.02 \\
RVECV & 23.5 & 24.42 & 43 & 0.001 \\
RVGCS & -14 & -11.48 & -6.3 & 0.002 \\
RCGCSE & 59.6 & 39.5 & 42.5 & 0.002 \\
RVGCSA & 49.9 & 44.2 & 16.1 & 0.2 \\
\hline
\end{tabular}

\subsection{Group I: HFpEF - Isolated Left Ventricle Type}

21 patients were seen in this group all of which showed normal systolic function of both left and right ventricle. The only positive imaging finding on CMR was increased E/GCSe'r of left ventricle with mean of 1.08 (1.03-1.1 95\% CI), (Figure 1) There was no correlation seen with NT pro BNP levels with $\mathrm{r}=-0.04$ (table 3 ) The mean LAVI and ECV in this group were $26.4 \mathrm{ml} / \mathrm{m}^{2}$ and $25.5 \%$ respectively.

\subsection{Group II: HFpEF-Isolated pcPH Type}

13 patients were seen in this group. All of these patients showed increased E/GCSe'r ratio with mean of 1.28 (1.01$1.5195 \% \mathrm{CI}$ ) which was higher than seen in group I patients difference being statistically significant $(\mathrm{p}<0.001)$. The LAVI was also increased with mean size of 30.38 (27.3-33.47 95\% CI) but did not show any significant correlation with E/GCSe'r $(r=0.12)$. was seen The right ventricle showed reduced systolic global circumferential and longitudinal strain with mean of $-11.48(-12.71$ to $-10.2495 \% \mathrm{CI})$ and -9.4 $(-13.5$ to $-8.495 \% \mathrm{CI})$ and reduced early diastolic strain rate with mean of 39.5 (16.1-62.2 95\% CI) both of which had a weak correlation with pro-BNP levels $(\mathrm{r}=-0.04)$ (Figures 2, 3). There were 3 patients in this group with history of coronary artery disease who had increased left ventricle ECV levels but no significant correlation was seen with ECV with E/GCSe'r. 

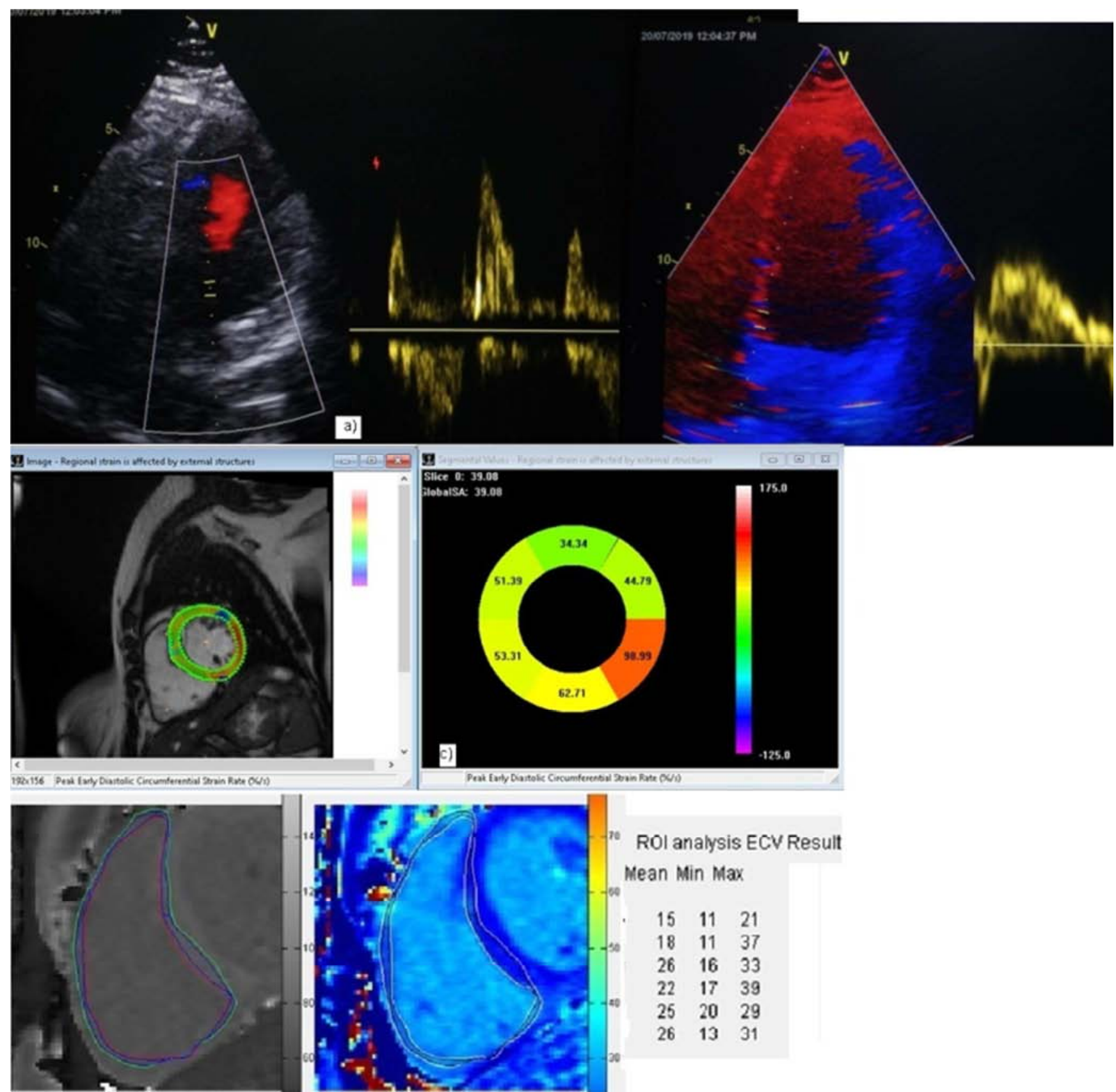

Figure 1. Group I patient of HFpEF-ilv phenotype with $a, b$ ) diastolic dysfunction on Doppler echocardiogram c) CMR strain analysis showing normal global circumferential strain of -18.5 d) reduced global circumferential strain early diastolic rate of 39.

\subsection{Group III: $\mathrm{HFpEF}$ with $\mathrm{CpcPH}$}

16 patients were seen in this group all which showed reduced E/GCSe'r with mean of $2.13(1.45-2.81,95 \% \mathrm{CI})$. The mean Right ventricle ECV was $43.0(39.5-46.5$ 95\%CI) while mean left ventricle ECV was $29.93(27.3-31.3495 \% \mathrm{CI})$ with a weak correlation with each other -0.53 (Figure 4). Marked reduction in right ventricle global circumferential and longitudinal strain was seen with mean of $-6.38(-10.68$ to $-2.0,95 \% \mathrm{CI})$ and $-5.5(-8.5$ to $-4.8 .95 \% \mathrm{CI})(\mathrm{r}=0.57)$. The right ventricle early diastolic strain rate was also reduced with mean of $42.6(34.7-50.495 \% \mathrm{CI})$. A moderate correlation of E/GCSe'r was seen with ECV of right ventricle $(\mathrm{r}=-0.44)$ (Figure 5). The mean LAVI was $34.71 \mathrm{ml} / \mathrm{m}^{2}$ (33.44-35.06 95\%CI) and showed weak correlation with E/GCSe'r and ECV ( $r$-0.09). Mean NT pro BNP levels in this group were $468 \mathrm{pg} / \mathrm{ml}$ and showed a strong correlation with both ECV and E/GCSe'r (r=0.74; p, 0.001) (Figure 6). 


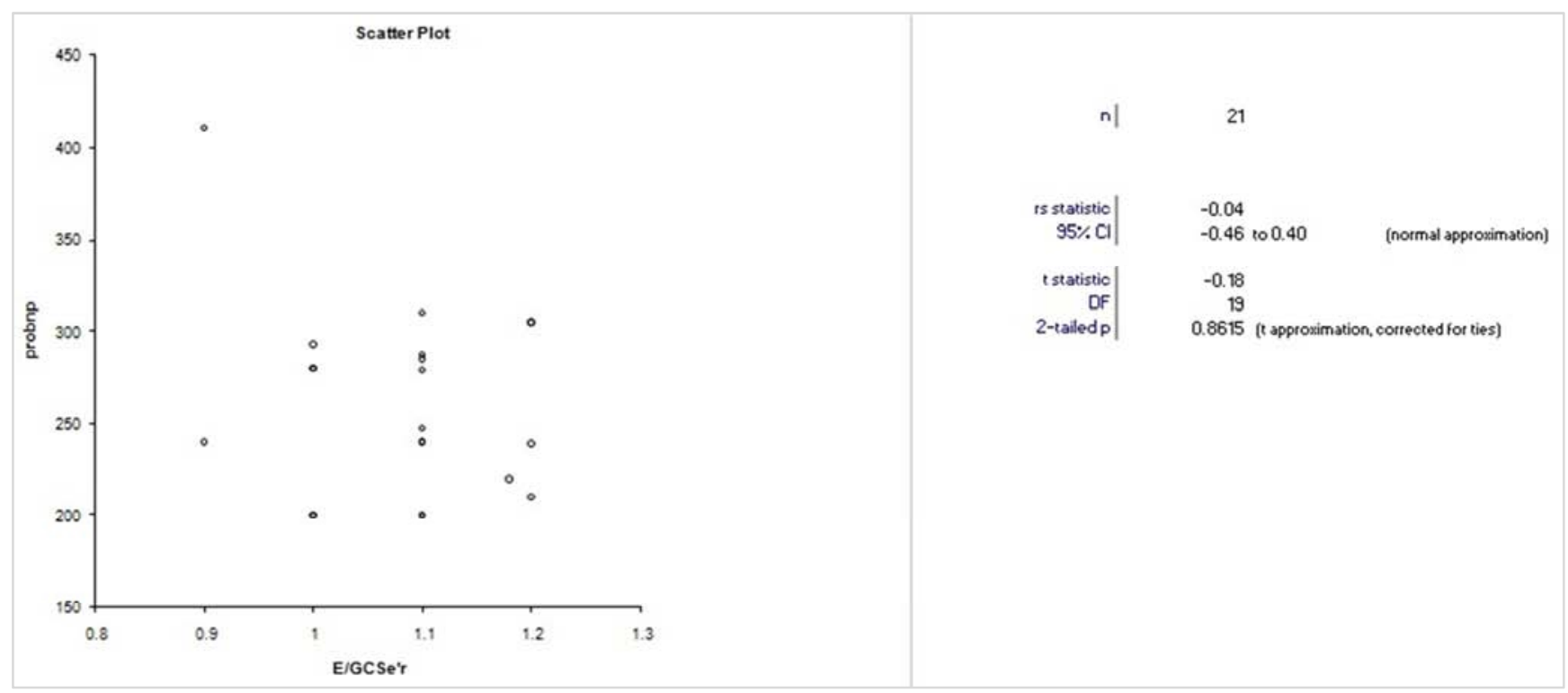

Figure 2. Scatter plot showing weak correlation of pro-BNP levels with GCSe'r in group I.

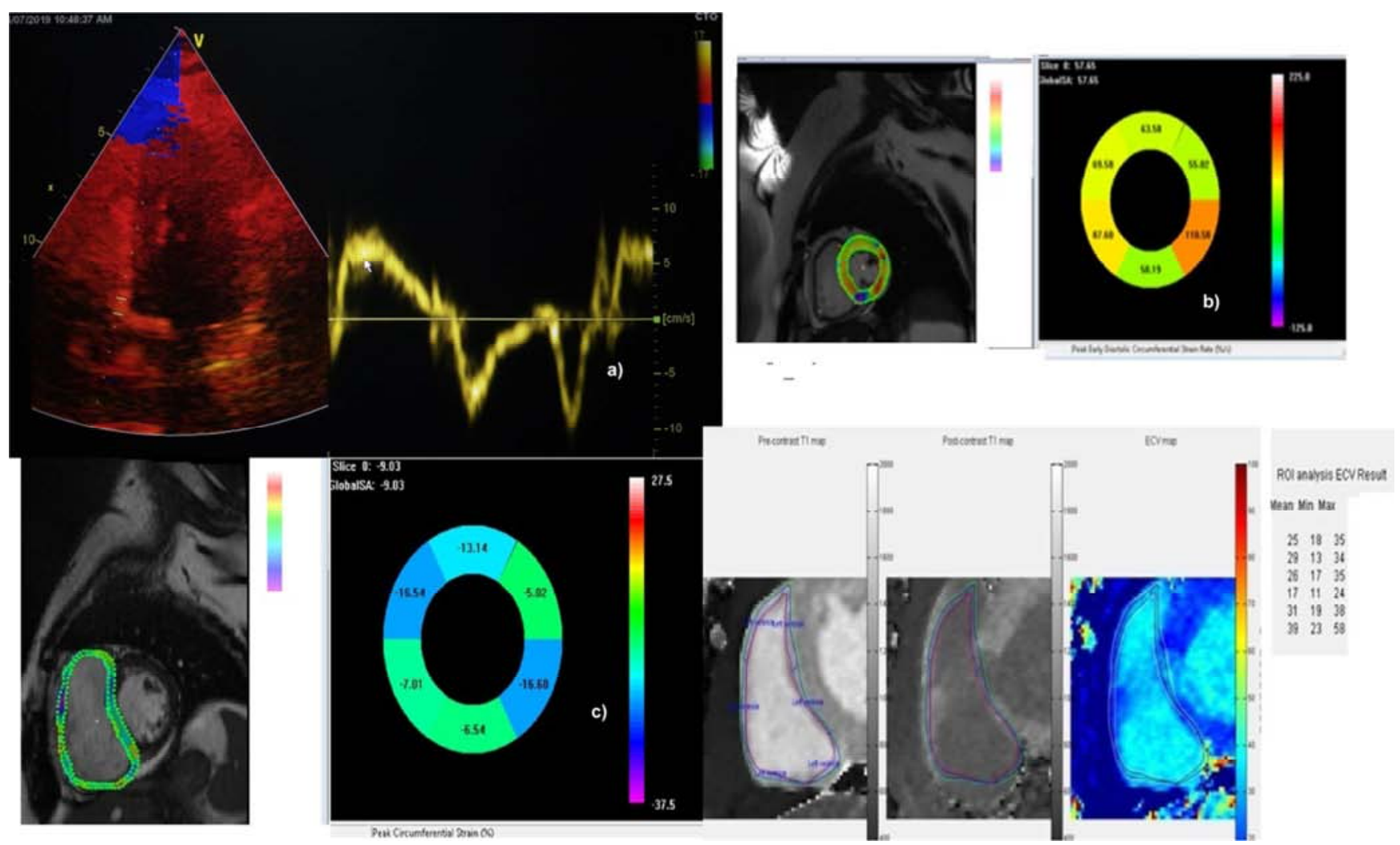

Figure 3. Group II patient of HFpEF-pcpH a) Diastolic dysfunction on tissue Doppler b) Left ventricle reduced early diastolic strain rate of 57 on CMR. C) CMR feature tracking showing right ventricle reduced global circumferential strain of -9.03 d) ECV of right ventricle showing mean ECV of $25 \%$. 


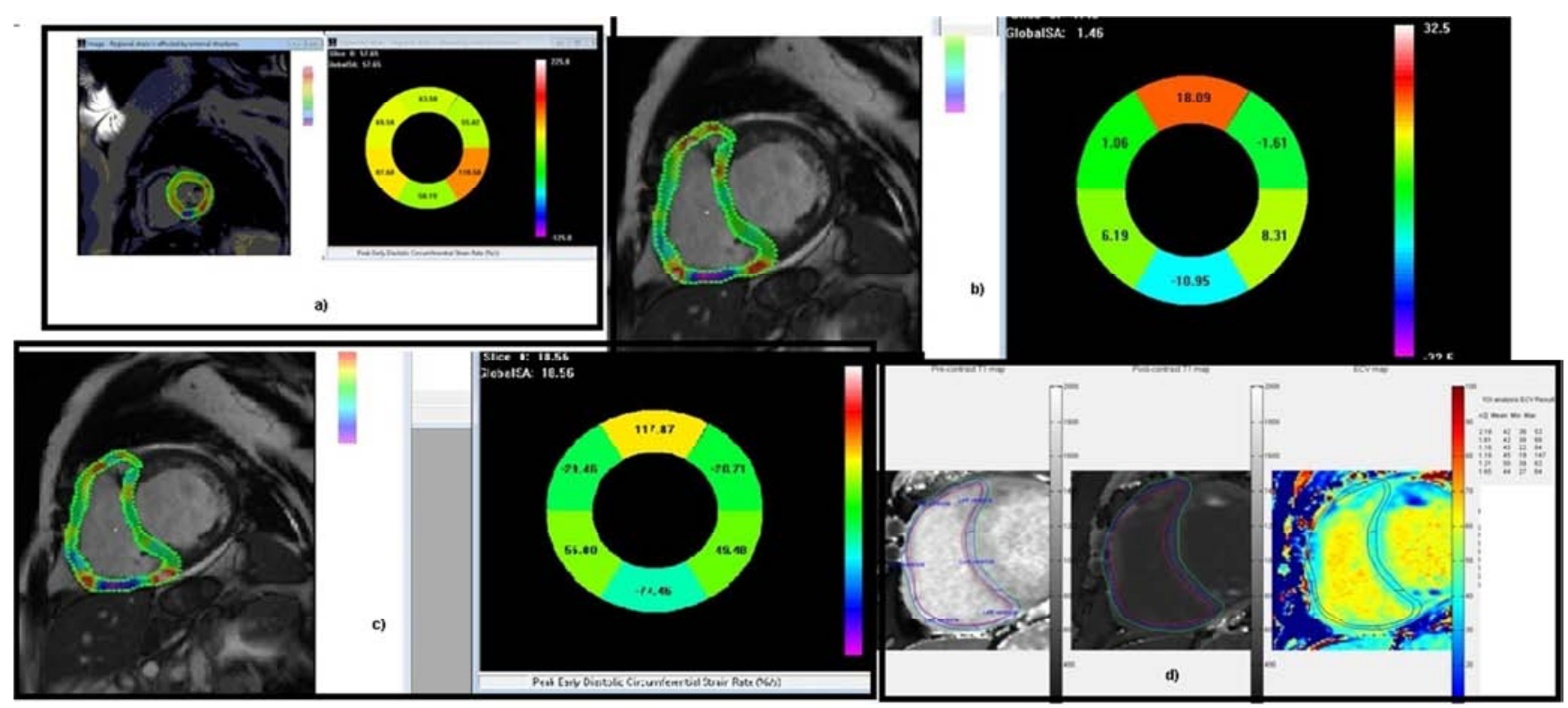

Figure 4. Group III HFpEF-CPCPH patient a) CMR showing reduced left ventricle GCSe'r of 57. b) CMR showing severely reduced right ventricle GCS of 1.4. and c) reduced right ventricle GCSe'r of 18.5. d) Mean ECV of right ventricle $45 \%$.

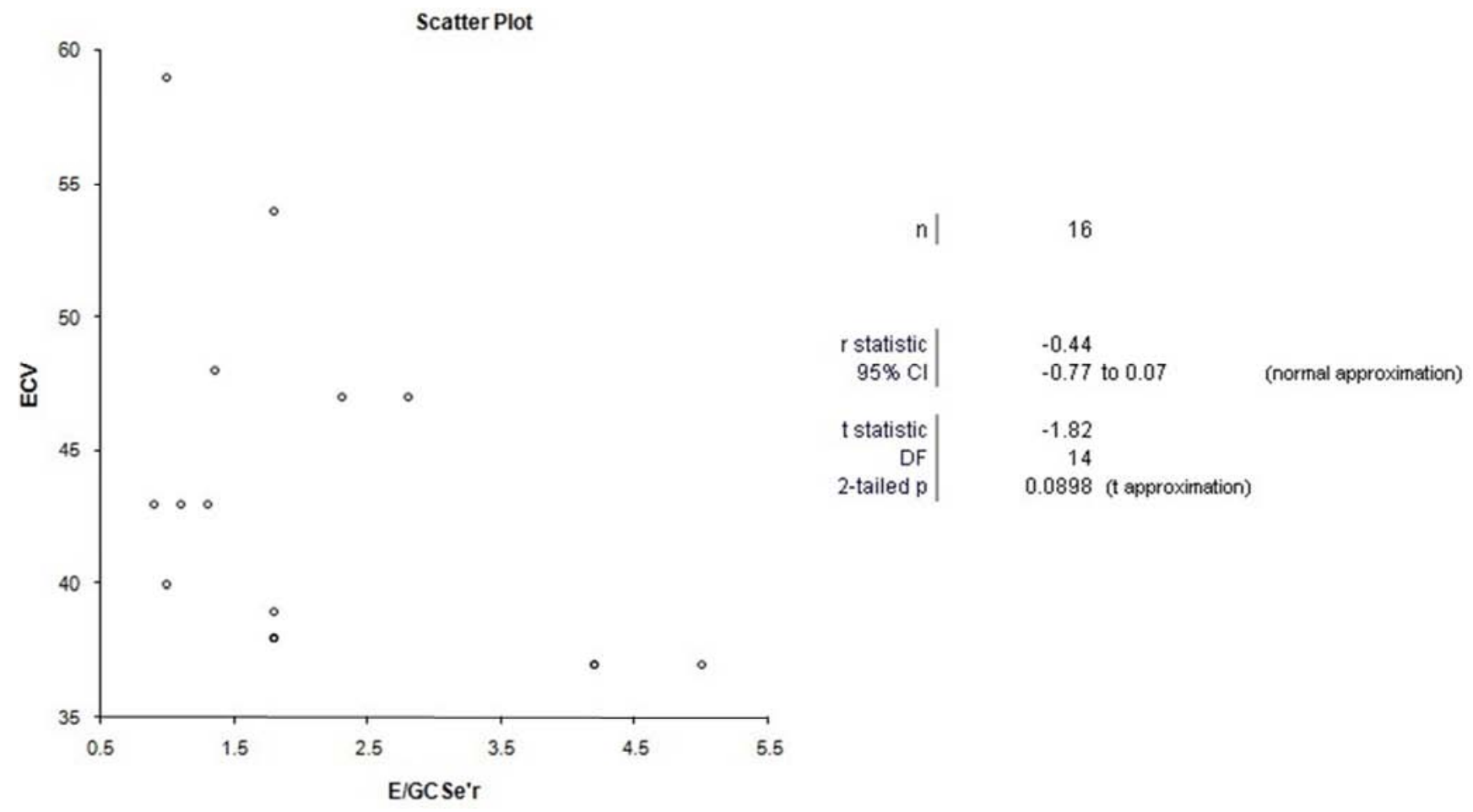

Figure 5. Scatter plot group III patient showing moderate correlation of E/GCSe'r and ECV. 


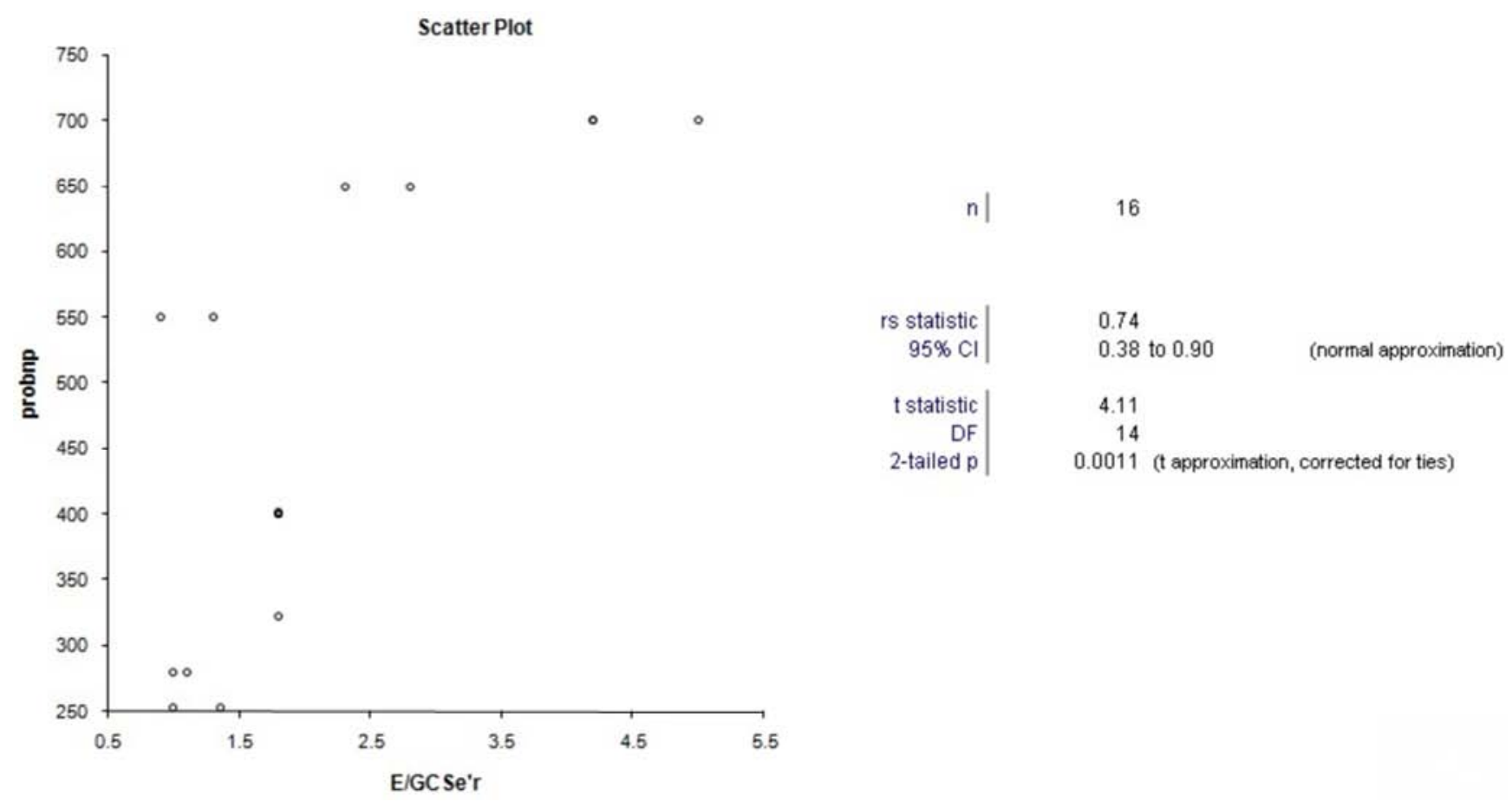

Figure 6. Scatter plot group III patient with strong correlation of E/GCSe'r with pro-NTBNP levels.

\section{Discussion}

In this study we retrospectively phenotyped patients of HFpEF by the use of CMR into three groups based on the changes in the structure and function of right ventricle which can have a direct bearing on the treatment and prognosis of these patients [6]. Etiologically HFpEF is an heterogenous group of disorder with a continuum of many pre existing diseases and hence it has been a challenge to categorise the disease state $[7,8]$. This study successfully classified HFpEF into three imaging phenotypes. Group I i.e those with isolated left ventricle type of $\mathrm{HFpEF}$ formed $21 \%$ patients and had no significant structural changes. Only positive parameter was increased E/GCSe'r ratio of left ventricle due to reduced left ventricle early diastolic strain rate. Patients of this imaging phenotype were those with preexisting morbidities like obesity, metabolic syndrome, diabetes, aging and menopausal females where there was uncoupling of ventricular filling and pressures as has been shown in various studies [9]. Also all these phenotypes are in the early stage of disease and also donot show any change in the ECV of left ventricle. This is contrary to the findings seen in patients with altered systolic functions or HFrEF patients where increased ECV forms a major determinant factor in etiopathogenesis and prognosis (9). The study also shows that no significant changes in LAVI were seen in this phenotype suggesting a lack of any significant structural change). The Health $\mathrm{ABC}$ trial [10] also showed patients with comorbidities form a separate community population and have a systemic inflammatory state which induces potent inflammatory cytokines such as IL-6 and tumor necrosis factor alpha (TNF- $\alpha$ ) which lead to diastolic dysfunction and elevated end diastolic pressures leading to HFpEF. Diagnosis of this phenotype of patients has a major implication on management and prognosis as correction of above comorbid factors can result in complete reversal of disease. Group II patients of HFpEF of Ipc PH type showed consistent elevation of left ventricle E/GCSe'r in the present study and also showed structural changes in the left ventricle, left atrium along with functional changes in systolic and diastolic function of right ventricle thereby suggesting back pressure effects of post capillary afterload. Pasha etal [5] also showed similar phenotypic changes by the use of CMR. Most of these patients had long standing systemic hypertension coupled with metabolic syndrome which lead to arterial stiffening, left ventricular hypertrophy and positive remodeling causing diastolic stiffening and structural changes in left ventricle [8]. This lead to higher left atrial pressure and contraction and eventual left atrial dilatation with these changes being eventually passed to post capillaries of pulmonary vasculature which also show increased pulmonary wedge pressures [11]. Even though screening echocardiography reveals structural changes of left atrial enlargement, Left ventricle hypertrophy and left ventricle diastolic dysfunction but is inadequate to detect IpcPH type of phenotype as it cannot determine pulmonary artery wedge capillary pressure [12]. With the use of feature tracking in CMR we evaluated the diastolic and systolic patterns of right ventricle as a measure of changes in the post capillary pulmonary venous system as a non invasive biomarker in this phenotype of HFpEF patients. Study showed a statistically reduced right ventricle systolic GCS, GLS and GCSe'r which also had a positive correlation with E/GCSe'r of left ventricle. Earlier diagnosis of this class of patients was only possible by using of right heart catheterisation. The present study shows that CMR due to higher spatial resolution when 
combined with feature tracking strain analysis can be used to detect this phenotype of HFpEF patients. This group of patients have alterations of biventricular hemodynamics and are very sensitive to fluid overloading and also have severe excerise intolerance, increased dysnoea on exertion [13, 14]. So detection of this phenotype is likely to lead to alterations in management with measures like use of diuretics and angiotensin blockers to reduce post capillary after load of right ventricle which improves their management and disease progression. Group III patients in the study i.e. those with HFpEF- CpcPH type formed the second largest group and comprised of patients who had already developed precapillary vasoconstrictive changes which lead to structural changes in right ventricle with increase in the ECV and wall thickness of right ventricle wall which further reduced systolic and diastolic right ventricle functions. It has been proposed that prolonged raised post capillary pressures leads to damage of the alveolar-capillary barrier (also known as alveolar-capillary stress failure) thus causing vasoconstricting changes which increases right ventricular after load triggering structural changes in right ventricle and failure and worsens the prognosis $[15,16]$. Increased ECV alongwith marked reduced right ventricle GCS and GLCSE were strong indicators to detect this group of patients of HFpEF. This group of patients also showed a high correlation with Pro BNP levels due to advanced stage of disease process alongwith grossly increased LAVI which was not seen in other two groups in the present study. These findings had a bearing on the management as well as prognosis as it would warrant the use of phosphodiesterase- 5 and endothelin inhibitors to control pulmonary vasculature changes in these patients.

The study has some limitations; namely small number of patients, this being an initial experience with the use of CMR in such patients. Second limitation has been the lack of invasive hemodynamic parameters of right heart catherisation.

\section{Conclusion}

HFpEF has become more prevalent than HFrEF worldwide. Its early diagnosis and management is quite challenging due to heterogenous nature of problem with a continuum of pre existing co morbidities. The study shows that with the use of modern imaging techniques like cardiac MR HFpEF can be classified into three imaging phenotypes. The commonest group of patients are those of IIV type i.e group I who have isolated left ventricle diastolic failure with altered GCSe'r and with preexisting comorbidities. Group III patients were the second commonest i.e those of HFpEF- CPCH type with pulmonary hypertension and had both structural and functional changes in right ventricle and showed right ventricle fibrosis with increased ECV and impaired systolic and diastolic right ventricle functions. Group II patients were HFpEF-pcPH type with left ventricle and right ventricle diastolic failure but with no structural change and fibrosis with right ventricular fluid overload.

E/GCSe'r of left ventricle alongwith right ventricle ECV were two strong biomarkers to determine the imaging phenotypes which can influence the management protocols and prognosis of these patients. To our knowledge this is the first study of use of CMR to classify imaging phenotypes of HFpEF using CMR.

\section{Abbreviations \\ CMR: Cardiac MR \\ ECV: extracellular volume \\ GCS: global circumferential strain \\ GLS: global longitudinal strain \\ GLSe'r: global longitudinal early diastolic strain rate}

\section{Conflict of Interest}

All the authors donot have any possible conflict of interest.

\section{Acknowledgements}

We acknowledge 1. Siemens healthineers Germany for Strain analysis software version 2.0 2. Einer Heiberg, Medviso, Lundh, Sweden for ECV estimation software for research purpose. 3. Mr Gurvinder singh for statistical analysis and manuscript preparation.

\section{References}

[1] Yancy CW, Jessup M, Bozkurt B, Butler J, Casey DE, Jr, Drazner $\mathrm{MH}$, et al. $2013 \mathrm{ACCF} / \mathrm{AHA}$ guideline for the management of heart failure: A report of the American College of Cardiology Foundation/American Heart Association Task Force on Practice Guidelines. Circulation. 2013; 128: 1810-52.

[2] Loehr LR, Rosamond WD, Chang PP, Folsom AR, Chambless LE. Heart failure incidence and survival (from the Atherosclerosis Risk in Communities study) Am J Cardiol. 2008; 101: 1016-22.

[3] Pieske B, Schöpe CT, Boer RA, Fraser AG, Anker SD, Donal E, Edelmann F, Fu M, Guazzi M etal. How to diagnose heart failure with preserved ejection fraction: the HFA-PEFF diagnostic algorithm: a consensus recommendation from the Heart Failure Association (HFA) of the European Society of Cardiology (ESC) European Heart Journal 2019; 40: 3297-3317.

[4] Samson R, Jaiswal A, Ennezat PV, Cassidy M; e Jemtel TL. Clinical Phenotypes in Heart Failure With Preserved Ejection Fraction. JAHA 2016; 115: 1-15.

[5] Pasha MC, Zhan Y, Debs D, Shah DJ. CMR in the evaluation of diastolic dysfunction and phenotyping HFpEF. JACC. Cardiovascular imaging 2020; 113: 283-296.

[6] Patel Rm, Li E, Brandon C, Stanley A, Benefield, Vincenzo B etal. Diffuse right ventricular fibrosis in heart failure with preserved ejection fraction and pulmonary hypertension. ESC Heart failure. 2020; 7: 254-264.

[7] Borlaug BA. The pathophysiology of heart failure with preserved ejection fraction Nat Rev Cardiol. 2014 Sep; 11 (9): 507-15. 
[8] Yancy CW, Jessup M, Bozkurt B, Butler J, Casey DE Jr, Drazner MH, Fonarow GC, Geraci SA et al, American College of Cardiology Foundation., American Heart Association Task Force on Practice Guidelines. J Am Coll Cardiol. 2013 Oct 15; 62 (16): e147-239.

[9] Mentz RJ, Kelly JP, von Lueder TG, Voors AA, Lam CS, Cowie MR, Kjeldsen K, Jankowska EA, Atar D, Butler J, Fiuzat M, Zannad F, Pitt B, O'Connor CM Non cardiac comorbidities in heart failure with reduced versus preserved ejection fraction. J Am Coll Cardiol. 2014 Dec 2; 64 (21): 2281-93.

[10] Kalogeropoulos A, Georgiopoulou V, Psaty BM, Rodondi N, Smith AL, Harrison DG, Liu Y, Hoffmann U, Bauer DC, Newman AB, Kritchevsky SB, Harris TB, Butler J, Health $\mathrm{ABC}$ Study Investigators. Inflammatory markers and incident heart failure risk in older adults: the Health $\mathrm{ABC}$ (Health, Aging, and Body Composition) study. J Am Coll Cardiol. 2010 May 11; 55 (19): 2129-37.

[11] Opitz CF, Hoeper MM, Gibbs JS, Kaemmerer H, Pepke-Zaba J, Coghlan JG, et al. Pre-capillary, combined, and postcapillary pulmonary hypertension: a pathophysiological continuum. J Am Coll Cardiol. 2016; 68 (4): 368-78.
[12] Redfield MM, Jacobsen SJ, Borlaug BA, Rodeheffer RJ, Kass D. Age- and gender-related ventricular-vascular stiffening: a community-based study. Circulation. 2005 Oct 11; 112 (15): 2254-62.

[13] Olsen FJ, Bertelsen L, de Knegt MC, et al. Multimodality cardiac imaging for the assessment of left atrial function and the association with atrial arrhythmias. Circ Cardiovasc Imaging 2016; 9: e00494.

[14] Lai YC, Potoka KC, Champion HC, Mora AL \& Gladwin MT (2014). Pulmonary arterial hypertension: the clinical syndrome. Circ Res 115, 115-130.

[15] Borlaug BA, Nishimura RA, Sorajja P, Lam CS \& Redfield MM (2010). Exercise hemodynamics enhance diagnosis of early heart failure with preserved ejection fraction. Circ Heart Fail 3, 588-595.

[16] Obokata M, Olson TP, Reddy YNV, Melenovsky V, Kane GC \& Borlaug BA (2018). Haemodynamics, dyspnoea, and pulmonary reserve in heart failure with preserved ejection fraction. Eur Heart J 39, 2810-2821.

[17] West JB \& Mathieu-Costello O (1995). Vulnerability of pulmonary capillaries in heart disease. Circulation 92, 622631. 